\title{
Self-assessment and Monitoring of CHI Performance in Digital Transformation
}

\author{
Rasa Bocyte $^{1(\otimes)}$ (D), Johan Oomen ${ }^{1}$ (D), and Fred Truyen ${ }^{2}$ (DD \\ 1 Netherlands Institute for Sound and Vision, Hilversum, The Netherlands \\ \{rbocyte, joomen\} @beeldengeluid.nl \\ 2 KU Leuven, Leuven, Belgium \\ fred.truyen@kuleuven. be
}

\begin{abstract}
To fully reap the benefits of digitisation and sustainably create value for their audiences, cultural heritage institutions (CHI) need to implement and monitor digital, data-driven strategies that touch upon all aspects of how organisations operate. This can range from staffing and skills development to adoption of metadata models, novel audience engagement approaches and methods for collecting and using user data. We introduce the concept for the CHI Self-Assessment Tool that enables institutions to assess their strategy and plan against several aspects of digital transformation. The tool proposes a novel approach on how CHIs can continuously gather data on their activities and use insights from this data to adjust their strategies and increase their digital maturity. Equally, this data can be used by policy-makers to implement more effective policies and support the sector with targeted capacity building.
\end{abstract}

Keywords: Digital transformation · Impact · Self-assessment

\section{Introduction}

Since the cultural heritage $(\mathrm{CH})$ sector started engaging with large-scale digitization projects, many institutions realized that they needed to implement integrated, data-driven digital workflows and strategies in order to make the most out of digital assets [6]. It soon became clear isolated projects with digital collections often result in momentary engagement and require deeper organizational and leadership changes to deliver sustainable impact to target communities [3,5]. The currently used concept of digital transformation tries to encapsulate this more comprehensive and integral digital approach. Europeana provides the following definition: "Digital transformation is both the process and the result of using digital technology to transform how an organisation operates and delivers value. It helps an organisation to thrive, fulfil its mission and meet the needs of its stakeholders" [2]. This definition implies rethinking of business models, value chains as well as participatory relationships between the CHIs and their audiences. Additionally, it highlights that digital transformation is an ongoing process with a constantly moving target rather than a set goal [4]. Thus data needs to be collected not only to define targets and track progress towards them but also to monitor trends and signals in the sector

(C) The Author(s) 2021

G. Berget et al. (Eds.): TPDL 2021, LNCS 12866, pp. 128-132, 2021.

https://doi.org/10.1007/978-3-030-86324-1_16 
and use them to further update digital strategies and inform decision-makers (funding bodies, European and national policy makers, networks and umbrella organisations) on appropriate actions.

The CHI Self-Assessment Tool (SAT) was conceived in this context to empower cultural heritage professionals and decision-makers to implement effective digital strategies. It presents a data-driven approach for enabling CHIs to continuously monitor their performance and become more confident in using insights from data to adjust their strategies and increase the impact of their activities. The innovation of the tool lies in its unique proposition to (1) iteratively gather data from a wide range of CHIs, (2) educate organizations on how such data can be translated into concrete actions, and (3) closely monitor trends in the sector that can inform decision makers regarding innovation, funding and capacity building needs. In this poster paper, we present the state of the art for monitoring and assessment activities in the heritage domain and describe the early concept of the SAT.

\section{State of the Art in Assessment and Monitoring Strategies}

Over the last few years, numerous online assessment and monitoring tools have been developed. We examined four examples that are widely adopted in the $\mathrm{CH}$ sector. All the tools start by asking users to fill out a survey of varying length and detail. The Tracker provides 93 questions divided into 12 areas, including Strategy \& Governance, Marketing \& Communications, and Finance \& Operations. Microsoft's Digital Transformation Framework for libraries and museums uses 16 questions to assess digital transformation across four areas: Enhanced Visitor Experience, Advanced Discovery, Dynamic Operations and Intelligent Environments. Both tools utilize rating systems to determine the current level of digital maturity and allow users to define target goals. The Digital Transformation Framework gives very specific examples for each level of maturity. For instance, one of the advanced levels in the Advanced Discovery area is "Computer vision and optical character recognition automatically generate additional metadata for realtime tagging". Such descriptions make this framework only applicable for very specific cases and provide a snapshot of the state of technology.

A scoring system is commonly used to present the results of the survey. Digitale Maturiteit uses a percentage from 1-100 to assess the digital maturity of each organization. Users are given a ranking in each category and can compare their performance with other organizations. Similarly, the Quick Innovation Scan used in the DEN Academy asks users to calculate their score out of a maximum of 20 points in four categories and to compare their results to a static chart. Such strict classification creates a false impression that all CHIs need to reach very specific goals and targets in order to succeed-it does not take into account the diversity of CHIs and the different paths available to them in order to reach digital maturity. This can be particularly discouraging for smaller organizations that target niche communities and do not see the need to offer the same services as internationally established CHIs.

All the examined tools provide insight about the status quo in an organization but do not offer concrete suggestions for follow up actions, hence their applicability is limited. For example, the Quick Innovation Scan offers generic guidelines such as "Seek 
opportunities to find collaboration partners inside and outside the organization to gain new knowledge and find new solutions". This can be useful to initiate a conversation about the necessary operational changes but more specific pointers are needed to ensure that appropriate measures and decisions are taken to achieve the envisioned targets, as well as make use of knowledge already available in the sector.

\section{CHI Self-assessment Tool}

Improving on the state of the art, the SAT is conceived as an interactive environment where $\mathrm{CH}$ professionals can collaboratively learn how to convert digital ambitions into digital strategies and continuously monitor their performance. The concept of the tool visualized in Fig. 1 presents its four components.

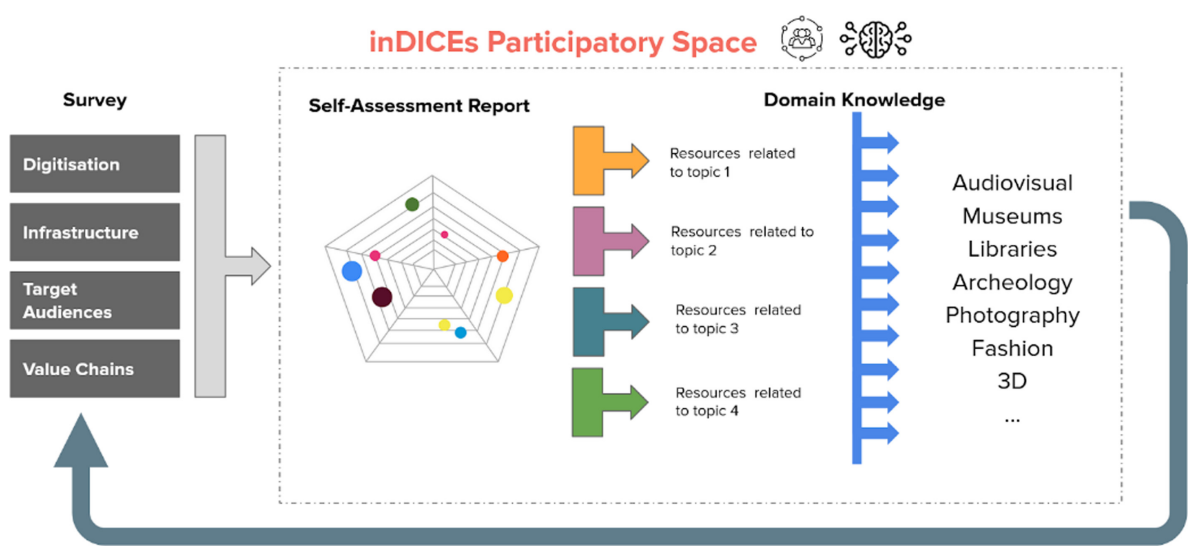

Fig. 1. CHI Self-assessment tool concept.

Survey. The users start by filling out a survey. In the first part, they are asked to answer general questions about their organization (size, target audiences, size and type of collections, participation in (inter)national networks, etc.) which help to situate each $\mathrm{CHI}$ in a specific context and domain. Based on these answers, the second part of the survey uses Likert scale questions to assess an organization's confidence with various aspects of digital transformation applicable to them, including adopted standards, skills and resources available in the organization, strategies for managing intellectual property rights and experience with online publication platforms. The survey sets out to give participants a rounded perspective of what digital transformation in the $\mathrm{CH}$ sector looks like and its many possible components. This is a learning experience in itself, and something that can be used for planning.

Self-assessment Report. The results of the survey are presented via data visualizations that showcase each $\mathrm{CHI}$ in relation to other organizations in the sector as well as focus on a particular domain or region/country. It is important to note that the report is not 
meant to give a rating or a grade on each question as this tends to favor larger, more advanced organizations with a wide outreach. Instead, the report is meant to highlight areas of high potential where further investment could lead to significant improvements. The aggregated results are available publicly for consultation by policy-makers and decision-makers to monitor the activities in the sector. All the examined already existing self-assessment tools stop at this stage.

Domain Knowledge. Based on the strengths and goals of each $\mathrm{CHI}$ as highlighted by the survey results, SAT provides targeted, domain-specific resources to support digital transformation processes (for instance, a media archive would be pointed to resources related to standards for audiovisual data enrichment). These resources have been either created specifically by the inDICEs project or aggregated from other web sources and $\mathrm{CH}$ communities. Additionally, SAT provides resources on what data collection strategies CHIs could use to monitor the impact of their activities and how to draw practical insights from this data. A complementary MOOC course will provide further guidance.

Participatory Space. SAT is embedded in the inDICEs Participatory Space that facilitates active engagement and knowledge sharing between $\mathrm{CH}$ professionals on topics related to digital transformation. It is specifically designed to engage participants in debates, brainstorming and community building activities. Again, a data-driven approach is used - topics discussed in the Participatory Space are visualized via embedded data visualizations that help to observe more granular trends in the sector. As new trends emerge, the initial survey questions are iteratively updated to reflect these changes.

As Fig. 1 indicates, SAT is meant to be used iteratively. Users receive notifications as new relevant resources are added and are encouraged to update the survey answer at regular intervals. Time-series data is used to visualize progress over time. Importantly, the SAT serves not only individual CHIs but also policy-makers who require monitoring tools to implement effective policies and capacity building activities [1]. Using the results of the SAT, they can monitor the effectiveness of their instruments over time and adjust them based on the data provided by the CHIs.

\section{Conclusions and Future Work}

This paper presented the concept of the CHI Self-Assessment Tool. During the conference, we will demonstrate the first prototype of the tool and present specific scenarios for its usage. Our further work will focus on testing this prototype with a diverse group of cultural heritage professionals as well as investigating strategies to ensure their sustained engagement with the tool.

Acknowledgements. This project has received funding from the European Union's Horizon 2020 research and innovation programme under grant agreement No 870792. 


\section{References}

1. Cerreta, M., di Girasole, E.G.: Towards heritage community assessment: indicators proposal for the self-evaluation in faro convention network process. Sustainability 12(23), 9862 (2016). https://doi.org/10.3390/su12239862

2. Europeana Pro, Building Digital Capacity. https://pro.europeana.eu/page/building-digital-cap acity\#step-2-defining-digital-transformation. Accessed 19 Apr 2021

3. James, D., Royston, C.: How to be a digital leader and advocate: the changing role of the digital department. MW2015: Museums and the Web 2015. https://mw2015.museumsandth eweb.com/paper/how-to-be-a-digital-leader-and-advocate-the-changing-role-of-the-digitaldepartment/

4. Ludden, J., Russick, J.: Digital Transformation: It's a Process and You Can Start Now. Museums and the Web 2020. https://mw20.museweb.net/paper/digital-transformation-its-a-process-andyou-can-start-now/

5. Malde, S., Kennedy, A., Parry, R.: Understanding the digital skills \& literacies of UK museum people-Phase Two Report (2019). https://doi.org/10.29311/2018.02

6. Terras, M., Coleman, S., Drost, S., et al.: The value of mass-digitised cultural heritage content in creative contexts. Big Data \& Society. January 2021 doi:https://doi.org/10.1177/205395172 11006165

Open Access This chapter is licensed under the terms of the Creative Commons Attribution 4.0 International License (http://creativecommons.org/licenses/by/4.0/), which permits use, sharing, adaptation, distribution and reproduction in any medium or format, as long as you give appropriate credit to the original author(s) and the source, provide a link to the Creative Commons license and indicate if changes were made.

The images or other third party material in this chapter are included in the chapter's Creative Commons license, unless indicated otherwise in a credit line to the material. If material is not included in the chapter's Creative Commons license and your intended use is not permitted by statutory regulation or exceeds the permitted use, you will need to obtain permission directly from the copyright holder.

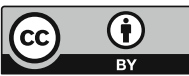

